\title{
MINIMUM VARIANCE INTERPOLATION AND SPECTRAL ANALYSIS
}

\author{
Kaushik Mahata and Damián Marelli \\ School of Electrical Engineering and Computer Science, University of Newcastle, \\ Callaghan, NSW 2308, Australia.
}

\begin{abstract}
Interpolation and spectral analysis of signals from finite number of samples is considered. When the observed data is of finite length, interpolation and spectral analysis of bandlimited signals using Shanon's framework leads to erroneous results. In spectral analysis this phenomenon is known as the spectral leakage problem. In this paper we address this issue from a minimum variance estimation perspective, and treat the generic case where the signal is not necessarily bandlimited. In contrast to traditional windowing based methods, the minimum variance framework leads to a convolutive transformation of the data, which employs a linear predictor. Simulations indicate a significant improvement in the performance.
\end{abstract}

\section{INTRODUCTION}

According to Shanon's sampling theorem, reconstructing a bandlimited signal $y(t)$ for any $t \in \mathbb{R}$, given $y(k), \forall k \in \mathbb{Z}$ requires computing the summation

$$
y(t)=\sum_{k=-\infty}^{\infty} y(k) \varphi(t-k),
$$

where $\varphi(t)$ is the sinc function i.e. its Fourier transform $\hat{\varphi}(\xi)$ is given by

$$
\hat{\varphi}(\xi)= \begin{cases}1, & |\xi| \leq 1 \\ 0, & |\xi|>1\end{cases}
$$

Clearly, the Fourier transform $\hat{y}(\xi)$ of $y(t)$ is assumed to satisfy

$$
\hat{y}(\xi)=0, \quad|\xi|>\pi
$$

Note that for existence of the Fourier transform $\hat{y}(\xi)$ we must have

$$
\int_{-\infty}^{\infty} y^{2}(t) \mathrm{d} t<\infty
$$

satisfied. Note that we assume that the sampling interval is unity, and this does not cause any loss of generality as it is always possible to adjust the time scale to satisfy this assumption.

Equation (1) can also be applied when $y(t)$ is a stationary stochastic process, see [1] and references therein, although (4)

\footnotetext{
The research is supported by the Australian Research Council.
}

does not hold in this case. Provided that the power spectral density of $y(t)$ is compactly supported in the interval $[-\pi, \pi]$, the equality (1) is formally given in form of a stochastic convergence: i.e.

$$
\lim _{n \rightarrow \infty} \sum_{k=-n}^{n} y(k) \varphi(t-k)=y(t), t \in \mathbb{R}
$$

both in mean squares and with probability one. However, the assumption that an infinite data sequence is available and can be processed via a computer is highly ideal assumption and does not hold in practice. In reality, the signal processing algorithm needs to deal with finite sample lengths. Suppose that we have access to $y(k)$ only for $k=0,1, \ldots, N-1$. Then the interpolation is often carried out by truncating the summation in (1):

$$
\bar{y}(t)=\sum_{k=0}^{N-1} y(k) \varphi(t-k)
$$

while the the Fourier transform of the reconstructed signal $y_{N}(t)$ is given by the discrete-time Fourier transform of $y(k), k=0,1, \ldots, N-1$, mutiplied by $\hat{\varphi}(\xi)$ :

$$
\hat{\bar{y}}(\xi)=\hat{\varphi}(\xi) \sum_{k=0}^{N-1} y(k) \mathrm{e}^{-\mathrm{i} \xi k} .
$$

The error introduced by truncating the summation is known as the spectral leakage error in the classical signal processing literature [2]. Observation over finite time window means a finite rectangular windowing in the time domain, which is equivalent to the convolution by a sinc function in frequency domain. This motivates the traditional way of dealing with the spectral leakage problem via windowing. The idea is to use an alternative window (like hamming window) such that the frequency response function of the time domain window has as small support as possible. Thereby, the error due to convolution is minimized. In this paper we consider an alternative point of view. We treat $y(t)$ as a continuous-time stochastic process, and pose the interpolation problem as the following estimation problem:

Let $y(t)$ be a stationary stochastic process. Given $t \in$ $\mathbb{R}$, and the samples $y(k), k=0,1, \ldots, N-1$ how can we 
construct a linear estimate $\tilde{y}(t)$ of $y(t)$ such that the mean square error $E\{y(t)-\tilde{y}(t)\}^{2}$ is minimized?

Note that the bandlimitedness is not assumed. The solution to the above problem is derived from the linear least squares filtering theory [3], which has some interesting properties. In contrast to the traditional windowing approach of dealing with the spectral leakage problem the minimum variance interpolation extends the observed data to the past and future via forward and backward filtering of the observed data through the optimal linear predictor. The resulting output is used for interpolation and spectral analysis. We also show an accurate method to computation. In the simulations we have shown the effectiveness of the proposed approach, where a significant improvement is observed compared to the traditional methods.

\section{MINIMUM VARIANCE INTERPOLATION}

\subsection{The fundamental result}

The answer to the question raised in the previous section comes from the theory of best linear estimation, and the solution is identical to that of the linear minimum estimation if the observed data comes from a Gaussian process. Let us denote the observed data vector as

$$
Y=\left[\begin{array}{llll}
y^{\prime}(n-1) & y^{\prime}(n-2) & \cdots & y^{\prime}(0)
\end{array}\right]^{\prime} .
$$

Note that $A^{\prime}$ denotes the transpose of $A$ in the sequel. In the sequel we assume $y(t) \in \mathbb{R}^{m}$, i.e. $y(t)$ can be a mutivariable stationary stochastic process. Then the best linear estimate of $y(t)$ that minimizes the mean square error is given by [3]

$$
\breve{y}(t)=C(t) Q^{-1} Y, \quad t \in \mathbb{R} .
$$

where

$$
Q:=\mathrm{E}\left\{Y Y^{\prime}\right\}, \quad C(t)=\mathrm{E}\left\{y(t) Y^{\prime}\right\} .
$$

Note that when $k \in\{0,1, \ldots, n-1\}$, then using the standard Matlab notation

$$
C(k)=Q((n-k-1) m+1:(n-k) m,:) .
$$

This imples after using in (9) that

$$
\breve{y}(k)=y(k), \quad k=0,1, \ldots, n-1,
$$

which is natural to expect.

Note that we assume that the inverse of $Q$ exists. When the inverse does not exist then we get a degenerate situation where $y(t)$ is a superposition of sinusoids [4]. In that case the rank of $Q$ gives the number of sinusiods in the mixture. There are many efficient ways to estimate the amplitudes, phases, and frequencies of the sinusoids from $Y$, see for example [4] and references therein. Hence a singular $Q$ leads to an elegant and well understood solution to the interpolation problem, and will not be considered in the sequel.
We assume $y(t)$ is a wide sense stationary process, and denote the covariance function by

$$
c(t)=\mathbf{E}\{y(s+t) y(s)\}, s, t \in \mathbb{R} .
$$

In the following we work on the expression (9) to explore the nature of the solution to the minimum variance interpolation problem.

Lemma 1 Let $\mathbb{S}$ be the set of all continuous-time functions of the form

$$
s(t)=\sum_{k=-\infty}^{\infty} c(t-k) u_{k}, \forall k, u_{k} \in \mathbb{R}^{m \times m} .
$$

Then the minimum variance interpolant $\breve{y}(t) \in \mathbb{S}$.

Proof: Note that

$$
C(t)=\left[\begin{array}{llll}
c(t-n+1) c(t-n+2) & \cdots & c(t)
\end{array}\right]
$$

Let $Q^{-1} Y=\left[\begin{array}{llll}u_{n-1}^{\prime} & \cdots & u_{1}^{\prime} & u_{0}^{\prime}\end{array}\right]^{\prime}$. Then the lemma follows direcly from (9).

The observation in Lemma 2.1 reveals a general structure which is supported by the bandlimited signals. In particular, we can express the signals in $\mathbb{S}$ in terms of a generic interpolating kernel in $\mathbb{S}$. In the rest of this paper we denote this interpolating kernel in $\mathbb{S}$ by $\varphi(t)$ (with a slight abuse of notation), and the reason behind this will be clear shortly. It is wellknown that

$$
\hat{\varphi}(\xi)=\hat{c}(\xi)\left[\sum_{k=-\infty}^{\infty} \hat{c}(\xi-2 k \pi)\right]^{-1}
$$

is the Fourier transform of the unique interpolating function in $\mathbb{S}$. Indeed it is readily verified using the Poisson summation formula and (11) that,

$$
\sum_{k=-\infty}^{\infty} \varphi(k) \mathrm{e}^{-\mathrm{i} \omega k}=\sum_{k=-\infty}^{\infty} \hat{\varphi}(\omega-2 \pi k)=I_{m}
$$

where $I_{m}$ is the $m \times m$ identity matrix. This in turn implies

$$
\begin{aligned}
& \varphi(0)=I_{m}, \\
& \varphi(k)=0, k= \pm 1, \pm 2, \ldots ;
\end{aligned}
$$

and also

$$
\hat{c}(\xi)=\hat{\varphi}(\xi)\left[\sum_{k=-\infty}^{\infty} \hat{c}(\xi-2 k \pi)\right],
$$

which after inverse Fourier transform gives

$$
c(t)=\sum_{k=-\infty}^{\infty} \varphi(t-k) c(k) .
$$

Note that if $\hat{c}(\xi)$ is bandlimited between $[-\pi, \pi]$, then (13) holds, with $\varphi(t)=\sin (\pi t) /(\pi t) I_{m}$. 
Remark 1 If $m=1$, i.e. $y(t)$ is a scalar signal then $\mathbb{S}$ is called a shift-invariant space which has played a fundamental role in the determininstic sampling theory [5]. This is remarkable that under the minimum variance framework the notion of $\mathbb{S}$ comes up naturally.

Using the previous result we now obtain an interpolation formula under the minimum variance interpolation framework, which resembles closely with the classical interpolation formula of Shanon.

Lemma 2 The linear minimum mean square estimator of $y(t)$ is given by

$$
\breve{y}(t)=\sum_{k=-\infty}^{\infty} \varphi(t-k) z_{k}
$$

where

$$
z_{k}=C(k) Q^{-1} Y, \quad k \in \mathbb{Z}
$$

Proof: Using (13) for any $\ell \in \mathbb{Z}$ we have

$$
\begin{aligned}
c(t-\ell) & =\sum_{k=-\infty}^{\infty} \varphi(t-\ell-k) c(k) \\
& =\sum_{k=-\infty}^{\infty} \varphi(t-k) c(k-\ell)
\end{aligned}
$$

Consequently, it follows that

$$
\begin{aligned}
C(t) & =[c(t-n+1) c(t-n+2) \cdots c(t)] \\
& =\sum_{k=-\infty}^{\infty} \varphi(t-k) C(k)
\end{aligned}
$$

Substituting (16) into (9) we get (14).

Note that from (10) and (14) it follows that

$$
z_{k}=\check{y}(k)=y(k), \quad k=0,1, \ldots, n-1 .
$$

Hence if we have access to the samples of $y(k)$ for all $k \in \mathbb{Z}$ then

$$
\check{y}(t)=\sum_{k=-\infty}^{\infty} \varphi(t-k) y(k) .
$$

If the power spectral density of $y(t)$ is bandlimited we recover the Shanon's interpolation formula, which shows the connection between the interpolation theory and the minimum variance interpolation theory. We note by passing that the availability of $y(k)$ for all $k \in \mathbb{Z}$ ensures $\check{y}(t)-y(t) \rightarrow 0$ with probability one when the signal is bandlimited. However, the perfect reconstruction property does not hold in general.
The Fourier transform of $\breve{y}(t)$ is the windowed discretetime Fourier transform of the sequence $z_{k}$ :

$$
\hat{\tilde{y}}(\xi)=\hat{\varphi}(\xi) \sum_{k=-\infty}^{\infty} z_{k} \mathrm{e}^{-\mathrm{i} \xi k}
$$

In summary what Lemma 2 suggests is the following. Instead of using $Y$, use a sequence $\left\{z_{k}: k \in \mathbb{Z}\right\}$ defined via (15) for interpolation and spectral analysis. The minimum variance estimate of the Fourier transform of $y(t)$ is the same as of the discrete-time Fourier transform of $z_{k}$. Similarly, the minimum variance estimate of $y(t)$ is obtained via the interpolating the sequence $z_{k}$ in (14), while the interpolating kernel $\varphi(t)$ depends on the covariance function of $y(t)$. The property of the sequence $z_{k}$ that supports the minimum variance property of the interpolated signal and the spectral estimator is that for any $k \in \mathbb{Z}, z_{k}$ is the best among all unbiased estimates of $y(k)$ which are linear in $Y$.

Now let us compare our findings with the windowing techniques used widely in many fields of digital signal processing. Unlike the traditional windowing techniques, here the linear transformation is essentially a linear filter. Now compare (18) with the standard DTFT based spectral estimator. Using (17) we have

$$
\hat{\bar{y}}(\xi)=\hat{\bar{y}}(\xi)+\hat{\varphi}(\xi)\left\{\sum_{k=-\infty}^{-1} z_{k} \mathrm{e}^{-\mathrm{i} \xi k}+\sum_{k=n}^{\infty} z_{k} \mathrm{e}^{-\mathrm{i} \xi k}\right\} .
$$

The second term in the right-hand-side of (19) is the correction term which accounts for the leakage error in an optimal way such that the variance of the estimation error associated to $\hat{\grave{y}}(\xi)$ is the minimum among all linear spectral analyzers of $y(t)$.

Computation of $\breve{y}(t)$ or $\hat{\tilde{y}}(\xi)$ requires the knowledge of the signal statistics, which are not known in general. In practice the user must estimate these statistics from the data $Y$. Hence question remains how can one compute the sequence $z_{k}$ from the data in a reliable way. This is addressed next.

\subsection{Using covariance models}

The fact that $\check{y}(t) \in \mathbb{S}$ lets us work with the sampled covariance function $c(k), k \in \mathbb{Z}$, and it is not required to know the complete the function $c(t)$ for every $t \in \mathbb{R}$. We must, however, know $\varphi(t)$. If the signal can be assumed to be bandlimited, then $\varphi(t)$ is known. If the signal is not bandlimited we can choose from a vast collection of interpolating kernels like cardinal splines, see $[6,7]$ and references therein. These functions have been proven to work satisfactorily in numerous prectical problems. In our case, we need to choose one which can interpolate the sampled covariances $c(k), k \in \mathbb{Z}$ in a satisfactory manner, while $c(k)$ can be estimated from $Y$. This is a significant simplification. But that is not sufficient. As $Y$ is of finite length we cannot estimate $c(k)$ for all $k \in \mathbb{Z}$. 
In fact, the estimate of $c(k)$ is not reliable when the magnitude of $k$ is above certain range depending on $n$. This problem is common and most statistical signal processing algorithms deal with it by estimating a parametric model of the covariance sequence, where the model can be parameterized by a finite number of parameters. The most popular approach to this end is to fit an autoregrassive (AR) or an autoregrassivemoving-average (ARMA) model to the data, where $c(k)$ is an exponentially decaying sequence. To be able to put the theory developed in the previous section in practice, we investigate how is it possible to incorporate an ARMA model of $c(k)$ in our framework.

Assumption 1 The covariance sequence $c(k), k \in \mathbb{Z}$ satisfies

$$
c(k)=H F^{k-1} G, \quad k=1,2, \ldots
$$

for some matrices $H, F, G$ with compatible dimensions. Furthermore, all the eigenvalues of $F$ are located inside the open unit disc in the complex plane.

In practice the user must estimate the matrices $H, F$ and $G$ from the data. There are several algorithms available to estimate these matrices reliably from the data. A fast possibility would be to use some form of subspace algorithms, which have very attractive computational and staistical properties. The subspace estimates are often used to initialize a maximum-likelihood algorithm to obtain an estimate with the optimal accuracy. Also the assumption of the eigenvelue of $F$ ensures that $\|c(k)\|_{2}$ does decay exponentially with increasing $|k|$. This is also natural to expect. In the sequel we assume that the matrices $H, F$ and $G$ are accurately known.

\section{Lemma 3 Under Assumption 1}

$$
z_{n+k-1}=H F^{k-1} x, \quad k=1,2, \ldots,
$$

and

$$
z_{-k}=G^{\prime}\left(F^{\prime}\right)^{k-1} \bar{x}, \quad k=1,2, \ldots
$$

where

$$
x=\left[\begin{array}{llll}
G & F G & \cdots & F^{n-1} G
\end{array}\right] Q^{-1} Y,
$$

and

$$
\bar{x}=\left[\begin{array}{llll}
\left(F^{\prime}\right)^{n-1} H^{\prime} & \left(F^{\prime}\right)^{n-2} H^{\prime} & \cdots & H^{\prime}
\end{array}\right] Q^{-1} Y .
$$

Proof: Consider $k \in\{1,2,3, \ldots\}$. Then under Assumption 1 we have

$$
\begin{aligned}
C(n+k-1) & =\left[\begin{array}{llll}
c(k) c(k+1) & \cdots & c(k+n-1)
\end{array}\right] \\
& =H^{k-1}\left[\begin{array}{llll}
G & F G & \cdots & F^{n-1} G
\end{array}\right] .
\end{aligned}
$$

Hence from (15) and (23) we get (21). Similarly it follows that

$$
\begin{aligned}
& C(-k)=\left[\begin{array}{llll}
c(-n-k+1) & c(-n-k+2) & \cdots & c(-k)
\end{array}\right] \\
& =G^{\prime}\left(F^{\prime}\right)^{k-1}\left[\left(F^{\prime}\right)^{n-1} H^{\prime}\left(F^{\prime}\right)^{n-2} H^{\prime} \cdots H^{\prime}\right] \text {, }
\end{aligned}
$$

yielding (22) when substituted in (15) and (24) is used.

Corollary 1 Under (l), the minimum variance sprectral analyser is given by

$$
\begin{aligned}
\hat{y}(\xi) & =\hat{y}(\xi)+\left\{\mathrm{e}^{-\mathrm{i} \xi(n-1)} H\left(\mathrm{e}^{\mathrm{i} \xi} I-F\right)^{-1} x\right. \\
& \left.+G^{\prime}\left(\mathrm{e}^{-\mathrm{i} \xi} I-F^{\prime}\right)^{-1} \bar{x}\right\} \hat{\varphi}(\xi) .
\end{aligned}
$$

Proof: Substitute (21) and (22) in (19). We have

$$
\begin{aligned}
\hat{y}(\xi)=\hat{y}(\xi)+\hat{\varphi}(\xi) & \sum_{k=1}^{\infty}\left\{\mathrm{e}^{\mathrm{i} \xi} G^{\prime}\left(F^{\prime} \mathrm{e}^{\mathrm{i} \xi}\right)^{k-1} \bar{x}\right. \\
& \left.+\mathrm{e}^{-\mathrm{i} \xi n} H\left(F \mathrm{e}^{-\mathrm{i} \xi}\right)^{k-1} x\right\},
\end{aligned}
$$

Since all the eigenvalues of $F$ are located inside the open unit disc in the complex plane, the summation in the right-hand side of the above equation converges yielding

$$
\begin{aligned}
\hat{y}(\xi)=\hat{\bar{y}}(\xi)+\hat{\varphi}(\xi)\left\{\mathrm{e}^{\mathrm{i} \xi} G^{\prime}\left(I-F^{\prime} \mathrm{e}^{\mathrm{i} \xi}\right)^{-1} \bar{x}\right. \\
\left.+\mathrm{e}^{-\mathrm{i} \xi n} H\left(I-F \mathrm{e}^{-\mathrm{i} \xi}\right)^{-1} x\right\},
\end{aligned}
$$

which upon simplification gives (25).

Lemma 3 and Corollary 1 gives us a nice closed form expression for the minimum variance spectral analyser in (25), which is straightforward to compute. There are two different ways to compute $\check{y}(t)$. Suppose that we want to upsample the sample sequence $\left\{y_{k}\right\}_{k=0}^{n-1}$ by a factor $M$. Then one defines

$$
\hat{y}_{\uparrow}(\xi)=\left\{\begin{array}{ccr}
\hat{\ddot{y}}(M \xi), & |\xi| \leq \pi / M \\
0, & \pi / M<|\xi| \leq \pi .
\end{array}\right.
$$

and samples $\hat{y}_{\uparrow}(\xi)$ over a sufficiently fine frequency grid. Subsequently we can compute the upsampled version of $\left\{y_{k}\right\}_{k=0}^{n-1}$ via the IFFT of the frequency domain samples of $\hat{y}_{\uparrow}(\xi)$. If the sampling grid in the frequency domain is sufficiently fine, we avoid any aliasing in time domain and the upsampled signal is accurately recovered. Alternatively, one can employ a purely time domain approach using (14), (17), (21) and (22). In this case to avoid increased leakage error one should compute $z_{k}$ for sufficiently large range of $k$. Computation of $x$ and $\bar{x}$ involves solving a potentially large system of equations, see (23) and (24). Nevertheless, there is a fast recursive way to compute $x$ and $\bar{x}$, which is closely related to the Kalman filtering. These recursions are given by the following Lemmata.

Lemma 4 Consider the sequence of vectors $\left\{\mathbf{x}_{k}\right\}_{k=0}^{n-1}$, which is given recursively as

$$
\mathbf{x}_{k}= \begin{cases}\left(F-K_{k-1} H\right) \mathbf{x}_{k-1}+K_{k-1} y(k-1), & k>0, \\ 0 & k=0 .\end{cases}
$$


The Kalman gain $K_{k}$ is given in terms of a positive definite matrix $T_{k}$ as

$$
K_{k}=\left(G-F T_{k} H^{\prime}\right)\left(c(0)-H T_{k} H^{\prime}\right)^{-1},
$$

where $T_{k}$ is recursively updated as

$$
T_{k}= \begin{cases}F T_{k-1} F^{\prime}+K_{k-1}\left(G^{\prime}-H T_{k-1} F^{\prime}\right), & k>0, \\ 0, & k=0 .\end{cases}
$$

Then it follows that

$$
x=\mathbf{x}_{n-1}, \quad E\left\{x x^{\prime}\right\}=T_{n-1} .
$$

\section{Proof: See [8].}

The recursions (26)-(28) are the Kalman filter recursions in somewhat nonstandard form. Here $\mathbf{x}_{k}$ is the Markovian state at time $k$ of the process associated to the unique inovations representation of the discrete-time data. In our context, $x=\mathbf{x}_{n-1}$ can be given a physical interpreatation. It is a sufficient statistic for optimally estimating the future values of $y(t), t>n-1$ from the observed data $Y$. In other words, as far as predicting the future values of $y(t)$ is concerned, all the relevant information present in $Y$ can be compressed in $x$. Similarly, as far as reconstruction of the past values of $y(t), t<0$ is concerned it is enough to know $\bar{x}$, which serve as a sufficient statistic. A result similar to Lemma 4 can also be given for recursive computation of $\bar{x}$. This recursion involves a very rarely used version of the Kalman filter, which runs in the backward direction. Although the proof of this result is not available to the best of our knowledge, it is intuitive, and is omitted for brevity.

Lemma 5 Consider the sequence of vectors $\left\{\overline{\mathbf{x}}_{k}\right\}_{k=0}^{n-1}$, which is given recursively as

$\overline{\mathbf{x}}_{k}= \begin{cases}\left(F^{\prime}-\bar{K}_{k-1} G^{\prime}\right) \overline{\mathbf{x}}_{k-1}+\bar{K}_{k-1} y(n-k+1), & k>0, \\ 0 & k=0,\end{cases}$

where the backward Kalman gain $\bar{K}_{k}$ is given in terms of a positive definite matrix $\bar{T}_{k}$ as

$$
\bar{K}_{k}=\left(H^{\prime}-F^{\prime} \bar{T}_{k} G\right)\left(c(0)-G^{\prime} \bar{T}_{k} G\right)^{-1},
$$

where $\bar{T}_{k}$ is recursively updated as

$$
\bar{T}_{k}= \begin{cases}F^{\prime} \bar{T}_{k-1} F+\bar{K}_{k-1}\left(H-G^{\prime} \bar{T}_{k-1} F\right), & k>0, \\ 0, & k=0 .\end{cases}
$$

Then it follows that

$$
\bar{x}=\overline{\mathbf{x}}_{n-1}, \quad E\left\{\bar{x} \bar{x}^{\prime}\right\}=\bar{T}_{n-1} .
$$

\section{SIMULATION RESULTS AND CONCLUSIONS}

We simulate $y(t)$ by passing a continuous-time white noise through the filter having a transfer function

$$
\frac{\lambda^{2} s+1.5 \lambda^{3}}{s^{3}+2 \lambda s^{2}+2 \lambda^{2} s+\lambda^{3}}
$$

When $\lambda=1$, the cut-off frequency of the filter is about 0.5 $\mathrm{Hz}$. By decreasing $\lambda$ the bandwith decreases. See [9] for the details about how such a process can be simulated. We sample the process with a sampling interval $0.1 \mathrm{sec}$, and decimate the signal further by a factor of 10 . Hence the decimated data has a sampling interval of $1 \mathrm{sec}$, which is then used to reconstruct the signal with a sampling interval $0.1 \mathrm{sec}$. We compare the performance of the proposed minimum variance interpolation (MVI) approach with the conventional truncated Shanon interpolation (TSI) in (8). Note that interpolation results using a window (say for example Hamming window) will be far worse than that would be obtained by TSI since the window distorts the data severely at the ends. Estimation results are presented in Table 1 where we compare the error energy as a fraction of the signal energy (NSR) for various values of $\lambda$. The order of the ARMA model used in MVI approach has been chosen by Akaike's information criterion [10]. In each simulation the signal is observed over a time interval of 1000 sec. Results in Table 1 clearly demonstrates the superiority of MVI over the traditional SI approach. It is also interesting

\begin{tabular}{|c|c|c|c|c|c|}
\hline & $\lambda=1$ & $\lambda=0.8$ & $\lambda=0.6$ & $\lambda=0.4$ & $\lambda=0.2$ \\
\hline MVI & $8.7 \mathrm{e}-3$ & $4.3 \mathrm{e}-3$ & $1.8 \mathrm{e}-3$ & $5.2 \mathrm{e}-4$ & $7.2 \mathrm{e}-5$ \\
\hline TSI & $8.9 \mathrm{e}-3$ & $4.5 \mathrm{e}-3$ & $2.0 \mathrm{e}-3$ & $8.3 \mathrm{e}-4$ & $4.1 \mathrm{e}-4$ \\
\hline
\end{tabular}

Table 1. NSR for different approaches a function of $\lambda$.

to note that the performance of the MVI approach improves significantly as the signal bandwidth $\lambda$ decreases.

Next we test the mimimum variance approach as a spectral analyser ${ }^{1}$. We consider a standard signal used frequently for this purpose:

$$
y(t)=\sin (t)+\sin (1.0571 t)+e(t)
$$

where $e(t)$ is a white noise with zero mean and variance $1 / 4$. The signal is sampled at a sampling frequency $1 \mathrm{~Hz}$, while 100 samples were collected. In such situations a spectral analyser is put to a test as it needs to resolve to closely spaced frequencies, and leakage errors can spoil the result significantly. The results from a typical realization is depicted in Figure 1 , where we compare the results ontained with the minimum variance spectral estimator with that obtained with other popular windowing techniques. A significant improvement is evident in the minimum variance case as the spectral lines associated with the sinusoids are very sharp. The frequencies of the

\footnotetext{
${ }^{1}$ Note that by spectral analyser we do not refer to an estimator of the power spectral density. Here a spectral analyser means an estimator of the Fourier transform of the signal
} 

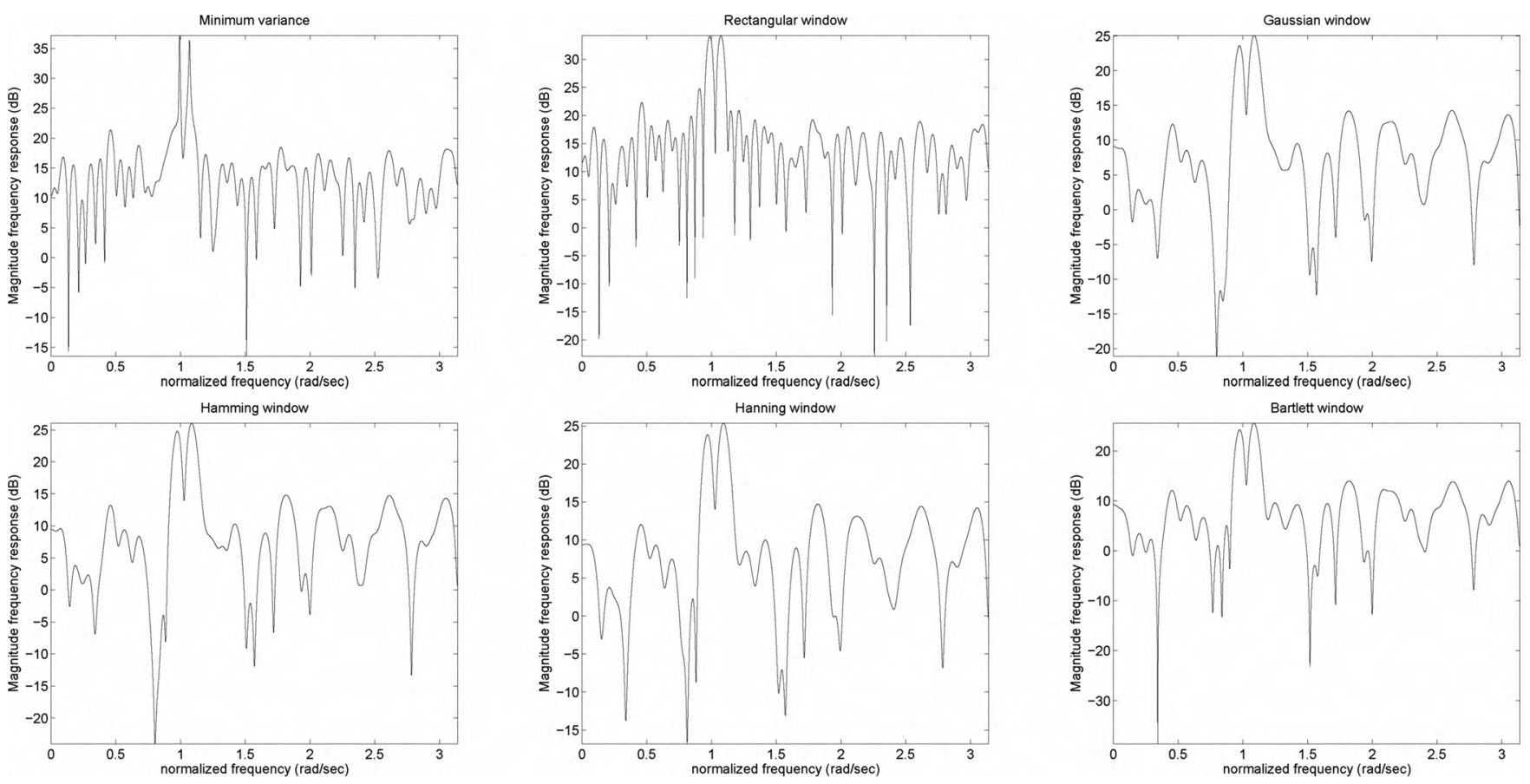

Fig. 1. The comparison between the magnitude of the frequency response function estimated by the minimum variance spectral estimator and that obtained via traditional windowing approaches for various wellknown windows. The underlying signal is described in (34).

sinusoids are identified accurately. In addition, the minimum variance spectral analyser does also accurately point out the existence of white noise in the spectrum. Note that all windows except the rectangular window lead to underestimation of the noise energy. It has been observed in the simulations that the performance of the proposed approach is somewhat sensitive to the underestimation of ARMA model order, while overestimation of the order does not cause major issues. For this reason, we use a model order somewhat higher than that returned by the Akaike's information criterion.

The minimum variance framework proposed in this paper brings in a significant improvement in the interpolation and spactral analysis performance. This approach is useful in numerous applications because of the fundamental nature of the underlying problem. For instance, we can use it in system identification and time-frequency analysis.

\section{REFERENCES}

[1] K. Seip, "A note on sampling of bandlimited stochastic processes," IEEE Transactions on Information Theory, vol. 36:5, pp. 1186-1186, 1990.

[2] A. V. Oppenheim and R. W. Schafer, Discrete-Time Signal Processing. Englewood Cliffs, New Jersey: Prentice Hall International, 1975.
[3] B. D. O. Anderson and J. B. Moore, Optimal Filtering. Englewood Cliffs, New Jersey: Prentice Hall, 1979.

[4] P. Stoica and R. Moses, Introduction to Spectral Analysis, 2nd ed. Prentice-Hall, Upper Saddle River, NJ, 2004.

[5] M. Unser, "Sampling-50 years after shanon," Proceedings of the IEEE, vol. 88:4, pp. 569-587, 2000.

[6] M. Unser and T. Blu, "Cardinal exponential splines: Part I-theory and filtering algorithms," IEEE Transactions on Signal Processing, vol. 53:4, pp. 1425-1438, 2005.

[7] M. Unser, "Cardinal exponential splines: Part II-think analog, act digital," IEEE Transactions on Signal Processing, vol. 53:4, pp. 1439-1450, 2005.

[8] L. H. Son and B. D. O. Anderson, "Design of Kalman filters using signal model output ststistics," Proceedings of IEE, vol. 120, no. 2, pp. 312-318, February 1973.

[9] T. Söderström, Discrete-time Stochastic Systems, 2 ed. London: Springer-Verlag, 2002.

[10] H. Akaike, "A new look at the statistical model identification," IEEE Transactions on Automatic Control, vol. 19:6, pp. 716-723, 1974. 